\title{
Optimizing resection of sessile serrated polyps
}

Removal of precancerous lesions during colonoscopy reduces colorectal cancer (CRC) incidence and mortality [1]. Recent data have highlighted that interval CRC occurs despite previous colonoscopy. Incomplete removal of polyps is one of the factors leading to interval CRCs [2]. Incomplete polypectomy of sessile serrated polyps (SSPs) is four-fold higher than that of conventional adenomas [3] and may contribute to a higher risk of interval CRCs.

Most SSPs are smaller than $2 \mathrm{~cm}$ (average size, 5-7 mm) [4] and can be removed safely and effectively at the time of routine colonoscopy. Optimal polypectomy technique varies depending on the individual characteristics of the polyp. SSPs often have a sessile or flat morphology with indistinct borders [5], which can make them more susceptible to incomplete removal. In light of the data showing an inordinately high incomplete polypectomy rate for SSPs, it is critically important for endoscopists to improve on the method of SSP removal.

We approach removal of suspected flat SSPs by using submucosal injection of dilute methylene blue with saline followed by snare electrocautery ( Figs. $1,2,3$ ). This technique provides both better visualization of the polyp borders and better positioning of the polypectomy snare to ensure complete resection. We believe this technique optimizes complete resection of flat SSPs, with the aim of reducing interval cancers. Further studies are needed to prove that the endoscopic and histologic recurrences are low and that a longterm reduction of interval cancers can be achieved using this technique.

Endoscopy_UCTN_Code_TTT_1AQ_2AD

Competing interests: None

\section{Seth Sweetser ${ }^{1}$, Todd H. Baron ${ }^{2}$}

${ }^{1}$ Division of Gastroenterology and Hepatology, Mayo Clinic College of Medicine, Rochester, Minnesota, USA

${ }^{2}$ Division of Gastroenterology and Hepatology, University of North Carolina School of Medicine, Chapel Hill, North Carolina, USA

\section{References}

1 Baxter NN, Warren JL, Barrett MJ et al. Association between colonoscopy and colorectal cancer mortality in a US cohort according to site of cancer and colonoscopist speciality. J Clin Oncol 2012; 30: 1570-1595

2 Farrar WD, Sawhney MS, Nelson DB et al. Colorectal cancers found after a complete colonoscopy. Clin Gastroenterol Hepatol 2006; 4: 1259-1264

3 Pohl H, Srivastava A, Bensen SP et al. Incomplete polyp resection during colonoscopy results of the Complete Adenoma Resection (CARE) study. Gastroenterology 2013; 144: 74-80e1

4 Higuchi T, Sugihara K, Jass JR. Demographic and pathological characteristics of serrated polyps of colorectum. Histopathology 2005; 47: $32-40$

5 Tadepalli US, Feihel D, Miller KM et al. A morphologic analysis of sessile serrated polyps observed during routine colonoscopy (with video). Gastrointest Endosc 2011; 74: $1360-1368$

\section{Bibliography}

Dol http://dx.doi.org/

10.1055/s-0034-1365379

Endoscopy 2014; 46: E231

(c) Georg Thieme Verlag KG

Stuttgart - New York

ISSN 0013-726X

Corresponding author

Seth Sweetser, MD

Mayo Clinic

200 First St. S. W.

Rochester, MN 55905

USA

sweetser.seth@mayo.edu

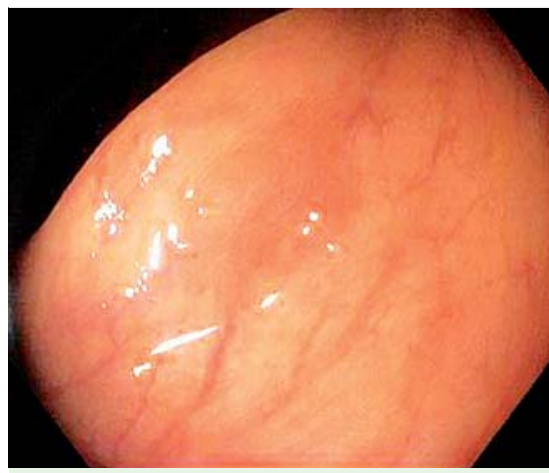

Fig. 1 Endoscopic white light view of 1-cm, flat, subtle sessile serrated polyp (SSP) with indistinct edges in ascending colon.

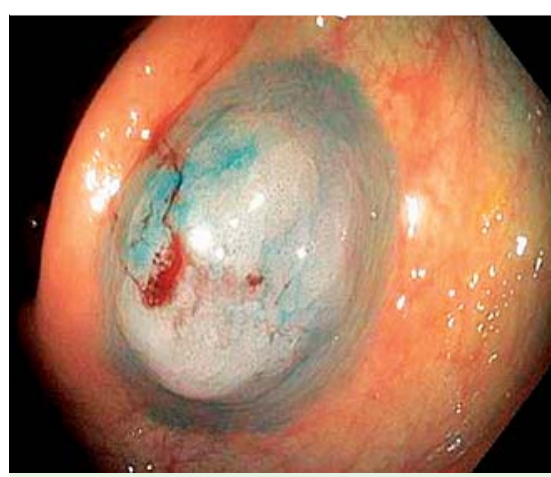

Fig. 2 Endoscopic view of SSP after submucosal injection of dilute methylene blue with normal saline. The edges of the SSP are now more clearly defined, allowing proper placement of the polypectomy snare.

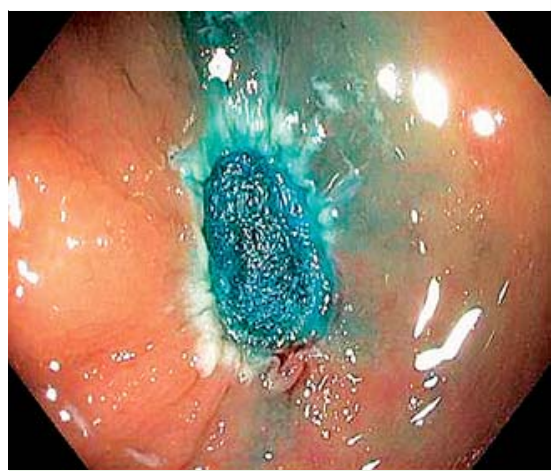

Fig. 3 Endoscopic view showing complete resection of the SSP with methylene-blue-stained muscularis propria. 